\title{
Correlação entre hemisfericidade e o aprendizado psicomotor em tarefas de comunicação gestuais
}

\author{
Artigo Original
}

\section{Sonia Yara Vallado}

Mestrado em Ciência da Motricidade Humana da UCB

soniavallado@ig.com.br

\section{Geane Pinto de O. Delgado.}

Mestrado em Ciência da Motricidade Humana da UCB

geanedelgado@terra.com.br

\section{Denise Oliveira Rezende de Souza}

Mestrado em Ciência da Motricidade Humana da UCB relaciones@uol.com.br

\section{Mario Roberto Guagliardi Jr}

Mestrado em Ciência da Motricidade Humana da UCB

mario@joianet.com.br

Vernon Furtado da Silva

Laboratório de Neuromotricidade II da Universidade Castelo Branco-RJ

vfs@castelobranco.br

Fernando Ribeiro Wanderley Lins

Mestrado em Ciência da Motricidade Humana da UCB

chinalins@ig.com.br

VALLADO, S.Y.; DELGADO, C.P.O.; SOUZA, D.O.R; GUAGLIARDI, M.R.J; SILVA, V.F.; LINS, F.R.W. Correlação entre hemisfericidade e o aprendizado psicomotor em tarefas de comunicação gestuais. Fitness \& Performance Journal, v.3, n.4, p.207-210, 2004.

Resumo: $\bigcirc$ objetivo deste estudo foi correlacionar ganhos de aprendizagem psicomotora, expressos em comunicação gestual (Linguagem de sinais), em escolares do ensino médio. Teoricamente, para que seja utilizada a comunicação gestual, especialmente na Língua de Sinais Brasileira (LIBRAS), de acordo com Moura (1993), cada um dos hemisférios cerebrais de uma pessoa ouvinte mostra uma decodificação predominantemente diferente. Sendo assim, no hemisfério esquerdo prevalecem funções lingüísticas, e no hemisfério direito prevalecem funções visuo-espaciais. A questão de pesquisa foi associada à possibilidade de que em casos de mono hemisfericidade esquerda, esta relação pudesse não ocorrer. Uma amostra de 100 alunos de ambos os gêneros, na faixa etária compreendida entre 15 e 17 anos, permitiu a identificação de 11 estudantes bi-hemisféricos (HB), 7 hemisféricitos esquerdo (HE) e 5 hemisféricitos direito (HD), sendo que tais identificações foram definidas pelo Teste de Movimento Conjugação Lateral dos Olhos (CLEM). Os componentes desses grupos foram submetidos posteriormente ao teste de sinais significativos de LIBRAS - um teste de sinais gestuais. Os dados obtidos foram tratados estatisticamente através de uma Análise de Variância, cujos resultados revelaram diferenças significativas em ganhos favoravelmente ao grupo mono hemisférico esquerdo, sendo a $£ 0,05$, como observado através do teste posterior de Tukey. Este resultado contraria a teoria da direcionalidade hemisférica, quando o fenômeno da hemisfericidade não éconsiderado.

Palavras-chave: Hemisfericidade, comunicação gestual, língua de sinais

Endereço para correspondência:

Rua Rainha Guilhermina, 131, apt 305 - Leblon - RJ - CEP 22441-120 
Hemispheric correlation with psychomotor learning in manual communication tasks

The objective of this study was to establish a correlation between hemispheres and the psychomotor learning in connection with the gesture communication. According to Moura (1993), when the gesture communication is practiced, especially in the Brazilian Sign Language (LIBRAS), each of the brain hemispheres of the listening person reveals a decode predominantly different. Therefore, left hemisphere prevails the linguistic functions, and right hemisphere the space-vision. In this survey we had the participation of one hundred students of both sexes, between 15 and 17 years old, with high school level attending one private school in the city of Rio de Janeiro. Once selected, the group was submitted to the CLEM TEST to identify the preference of the hemisphere processing. The students were divided in three hemispheric groups, that is, the right hemisphere $(H R)$, the left hemisphere $(H L)$ and the bi- hemispheres (HRL) .The components of each group were then submitted to a individual selective test including significative competency in relation to the communicative gestures signs. Analysis of variance was performed between the three groups $(p<0,05)$. The especific sign, regarding the performance of each student, indicated evidences that the linguistics aspects are preferably processed in the left hemisphere.

Keywords: Hemysphericy, gesture communication, Brazilian Sign Language (LIBRAS)
Correlacion entre hemisfericidad y el aprendisaje psicomotor em las tareas de comunicación gestual

El objetivo de este estudio fué correlacionar ganos del aprendisaje psicomotor expresados en la comunicación gestual ( Lenguaje de Señales ) en escolares de enseñanza media. Teoricamente, para que sea usada la comunicación gestual, especialmente en la Lengua de Señales Brasileña (LIBRAS), de acuerdo con Moura (1993), cada uno delos hemisferios cerebrales de una persona ojente muestra una codificación predominantemente diferente Siendo asi, en el hemisferio izquierdo predominan funciones linguisticas y en el hemisfério derecho prevalecen las funciones visuales -espaciales. El problema da la investigación fué asociada a posibilidade de que en casos de monohemisfericidade izquierda, esta relación pudiese no ocurrir. Una muestra de 100 alumnos de ambos os sexos, em las edades comprendidas entre 15 y 17 años, permitió la identificación de 11 estudiantes bi-hemisféricos $(\mathrm{HB}), 7$ hemisféricos izquierdos $(\mathrm{HE})$ y 5 hemisférios derechos $(\mathrm{HD})$, siendo que tales identificaciones fueron definidas por el Test de Movimiento en la Conjugación Lateral de los Ojos (CLEM). Los componentes de estos grupos fueron sometidos posteriormente al test de señales significativas de LIBRAS. Los datos obtenidos fueron tratados estadísticamente a través de un Analisis de Variasa, cujos resultados revelaron diferencias significativas en ganos favorablemente al grupo mono hemisferico izquierdo siendo $/ p \leq 0,05$, como observado através del test posterior de Tukey. Este resultado se opone a la teoria de la direccionalidad hemisferica cuando el fenomeno da la hemisferidad no es considerado.

Palabras clave: Hemisfericidad, comunicación gestual, Lengua de Señales Brasileña_(LIBRA)

\section{INTRODUCÃO}

O cérebro é dividido em duas partes, sendo estas denominadas hemisférios. De um modo geral, o hemisfério esquerdo controla a metade direita do corpo, e vice-versa, em razão do cruzamento das fibras nervosas na região correspondente ao tronco encefálico. Os hemisférios cerebrais aparentemente são iguais, mas, na realidade, têm diferenças anatômicas, neuroquímicas, funcionais, que, de certa forma, estão definidas geneticamente, mas que se aprimoram com a maturação, com a interação com o meio, proporcionando assim a especialização de cada hemisfério. Outras assimetrias também podem estar presentes em cérebros humanos (BRODAL, 1984).

No presente estudo, entendemos como hemisfericidade cerebral a acentuada tendência do processamento de informações de ser representado em um dos hemisférios, independente da especificidade do indivíduo. Ou seja, é a capacidade superior de um dos hemisférios sobre o outro, para a execução de determinadas funções.

Na maioria das pessoas, a área de Broca está localizada na área cortical do lobo frontal do hemisfério esquerdo, sendo responsável pela compreensão da linguagem e pela produção da fala. A área de Broca realiza o planejamento dos movimentos para a produção da linguagem falada e também a organização gramatical. A área de Wernicle é responsável pela compreensão da linguagem falada e está localizada na área cortical parieto-temporal do lobo esquerdo (CUPELLO E MIRANDA, 2003).

Com referência às funções lingüísticas, a dominância é determinada geneticamente, mas a preferência manual pode, pelo menos em parte, ser determinada pelo meio ambiente. Também é possível se afirmar que o hemisfério esquerdo está relacionado com as habilidades verbais, enquanto as habilidades não verbais dependem mais do hemisfério direito (BRODAL, 1984).

A hemisfericidade cerebral está relacionada não só aos fatores genéticos, maturativos, neuroquímicos já citados, mas também a outras influências, como posturas assimétricas durante o período pré-natal. Estas assimetrias posturais favorecem processamentos hemisféricos diferenciados, tanto perceptivos como motores. Dois terços dos fetos no terceiro trimestre da gestação estão posicionados com o seu lado direito externamente. Estimulação sensorial lateralizada durante o desenvolvimento pré e pós-natal pode determinar assimetrias cerebrais (FERNANDES, 2003).

Os fetos do sexo masculino têm um hemisfério cerebral direito maior do que o hemisfério cerebral esquerdo. Além disso, há evidências de que o cérebro masculino possa ser, na média, mais lateralizado ou assimétrico do que o feminino. Essas e outras diferenças na organização cerebral podem estar subjacentes a diferenças entre os gêneros, como, por exemplo, a maturação mais precoce das mulheres, o melhor desempenho das mulheres em tarefas lingüísticas, o melhor desempenho dos homens em tarefas visuo-espaciais, e a maior incidência de canhotos entre os homens.

O sistema nervoso do neonato já está bem desenvolvido, o que permite à criança, gradualmente, com a maturação, adquirir o sentido de direcionalidade, temporalidade, ritmicidade e lateralidade e a coordenação motora .

De acordo com Goleman, citado por Fernandes (2001), a partir dos 5-6 anos de idade, os hemisférios apresentam relativa plas- 
Tabela 1 - Apresentação das médias dos grupos e hemisféricos, desvio-padrão e testes posteriores respectivos.

\begin{tabular}{lccc}
\hline Grupos hemisféricos & Média & DP & Teste posterior (Tukey) Sig. \\
\hline HD (G1) & 4.60 & 1,34 & $\mathrm{HD} \times \mathrm{HE}(0.04)$ e BH $(0.004)$ \\
HE (G2) & 6.50 & 1.04 & $\mathrm{HE} \times \mathrm{HD}(0,04)$ e BH(0.689) \\
BH (G3) & 7.00 & 1.18 & $\mathrm{BH} \times \mathrm{HD}(0,004)$ e HE(0.689) \\
\hline
\end{tabular}

ticidade em sua habilidade para desenvolver diferentes funções, e só então começam a se especializar.

As funções simbólicas predominam sobre as motoras, a partir do décimo ano de vida. É durante a infância que a lateralização consolida os processos específicos de habilidades, dentro dos hemisférios cerebrais esquerdo e direito, considerando-se uma população normal (FERNANDES, 2001).

Atualmente, temos muitas formas de comunicação através de sinais visuais, como, por exemplo, a sinalização homógrafa, usada para a comunicação naval; a comunicação através de sinais nos aeroportos, no trânsito etc. A Língua Brasileira de Sinais (LIBRAS), língua materna dos indivíduos portadores de surdez, é uma das formas de comunicação que se realiza visualmente, através de movimentos coordenados, estruturação espaço-temporal, e é, ao mesmo tempo, uma forma simbólica de comunicação, apresentando conotações emocionais que são transmitidas através de expressões faciais e corporais.

A Língua de Sinais apresenta características para as quais cada um dos hemisférios cerebrais de pessoas ouvintes desenvolve características hemisféricas diferentes. Considerando os conceitos já mencionados, presentes em indivíduos com processamentos hemisféricos diferentes, este trabalho objetiva verificar, nos indivíduos já selecionados, através da aplicação do Teste CLEM, como hemisféricitos direito, esquerdo e bi-hemisféricitos, qual a diferença existente nos processamentos envolvendo uma comunicação por sinais visuais. Para esta análise, recorremos a alguns sinais utilizados na Língua de Sinais Brasileira, a qual engloba funções lingüísticas, e como tal, realizada preferencialmente por indivíduos hemisféricitos esquerdos; ao mesmo tempo, esta comunicação requer não só a decodificação percepto-visual, a transposição do visual para movimentos corporais manuais e expressivos, utilizando a percepção espacial, a relação espacial, movimentos seqüenciais rítmicos e memória, sendo estes aspectos mais pertinentes ao hemisfério direito. Considerando os conceitos já expostos, questionamos qual é o hemisfério que melhor processa funções lingüísticas mais relacionadas com o hemisfério esquerdo, e funções visuo-espaciais, mais próprias do hemisfério direito, ou ambos os hemisférios participam em igualdade.

\section{MÉTODO}

\section{Amostra}

Após a aplicação do Teste CLEM em 100 indivíduos, selecionando-os como HD, HE e BH, todos na faixa etária entre 15 e 17 anos, foi realizado o estudo com referência ao aprendizado da LIBRAS, com a participação de 23 indivíduos.

\section{INSTRUMENTOS}

\section{Teste de CLEM}

O Teste de Movimento Conjugação Lateral dos Olhos (CLEM) é correlacionado por parâmetros científicos com índices altamente significativos, comparados aos resultados obtidos através da eletroencefalografia e a tomografia por emissão de prótons (FAIRWEATHER E SIDAWAY, 1993), cujo objetivo é detectar as tendências de preferência de processamento hemisférico de um indivíduo.

Alguns cuidados na aplicação do teste foram tomados, como, por exemplo, aplicação individual em condições físicas, mentais e emocionais adequadas aos procedimentos e objetivo da testagem, evitando dessa forma distrações visuais e auditivas.

presente teste foi aplicado com o indivíduo sentado, dentro de uma cabine própria. Foi realizada uma filmagem da direção dos movimentos dos olhos, relacionando questões pertinentes à natureza de cada hemisfério, ou relativamente à natureza bihemisférica do indivíduo.

Foram selecionadas e aplicadas perguntas cujo objetivo era examinar a capacitação hemisférica direita. A reação contralateral dos olhos foi registrada pela filmadora e analisada durante o processamento de sua resposta. Os resultados foram aferidos através das imagens registradas em uma fita de vídeo, assinalando também o movimento do olhar nas fichas contendo as figuras tipo "face de relógio", conforme Borg (1983), citado por Fairweather e Sidaway (1993). Posteriormente, foi feita uma

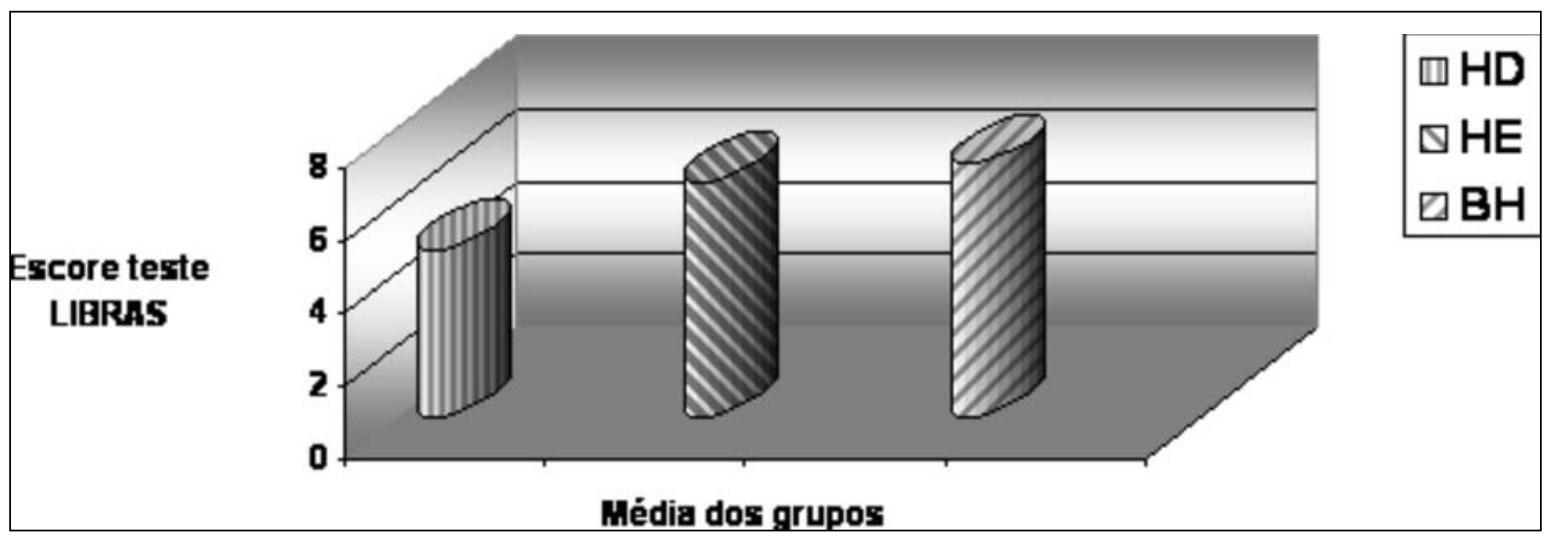

Figura 1 - Plotagem dos escores dos grupos hemisféricos correspondentemente ao teste da linguagem LIBRAS 
análise entre os registros das imagens e dos cartões, para garantir a fidedignidade dos resultados.

\section{Lista de sinais}

Para a verificação da capacidade diferenciada de reprodução, compreensão dos sinais envolvendo posições espaciais, direcionalidade, movimentos seqüenciais praxia fina, foi utilizada uma lista de dez sinais da LIBRAS. Os seguintes sinais foram utilizados: bom dia, obrigado, difícil, trabalho, homem, perguntar, amigo, cachorro, nascer, branco.

\section{PROCEDIMENTOS}

A pesquisa foi realizada individualmente, no turno da manhã, em sala com boa luminosidade e silenciosa. Os indivíduos participantes, iá selecionados como hemisféricitos diferenciados (HD, $\mathrm{HE}$ e $\mathrm{BH})$, inicialmente receberam a explicação sobre a natureza da pesquisa. Questionou-se também o conhecimento prévio dos sinais da LIBRAS. Só participaram os que não conheciam os mesmos. Foi realizado um treino individual, com a apresentação de cada sinal, por duas vezes, pelo pesquisador, e reprodução, por duas vezes, pelo participante. Após este treino, os dez sinais foram reproduzidos em seqüência pelo pesquisador. Finalmente, foi solicitada ao participante a reprodução um a um, à medida que cada sinal era nomeado pelo pesquisador.

\section{Avaliação}

Foram contabilizados como acertos a reprodução exata dos sinais testados, tanto em relação aos aspectos visuo-espaciais, à coordenação dos movimentos, à sua direcionalidade e ao significado lingüístico dos mesmos.

\section{Tratamento estatístico}

Os dados oriundos dos resultados da apresentação dos sinais da LIBRAS foram estudados através de uma Análise de Variância modelo Oneway, com testes posteriores de Tukey, para se definir a direção das possíveis diferenças entre grupos. Também foi utilizada estatística descritiva, com médias e desvios mostrados em tabelas comparativas. Para o teste da hipótese principal, utilizouse a probabilidade de erro alpha $=$ ou - de 0.05 .

\section{RESULTADOS E DISCUSSÃO}

Conforme explicado anteriormente, os resultados médios de reprodução e aprendizagem dos sinais da LIBRAS foram plotados, sob forma comparativa, na Tabela 1 .
Para uma melhor visualização do resultado acima, o mesmo foi plotado na figura 1. Observar na referida figura, as diferenças produzidas em referência à característica hemisférica de cada grupo.

Os resultados da Análise de Variância utilizada no estudo dos dados dos testes indicaram haver significância estatística, conforme mostrado na Tabela 1. Ou seja, com F(2,19) 77,14; a $<0,05$. Utilizando-se os testes posteriores de Tukey, ficou evidente que o grupo de indivíduos bi-hemiféricitos foi significativamente superior em performance do que o grupo hemisféricito direito, e também superior ao grupo hemisféricito esquerdo, todavia, neste caso, sem significância considerável. Também revelou aquela análise detalhada que os hemisféricitos esquerdos foram melhores que os indivíduos hemisféricitos direitos.

Foi possível concluir, após a comparação dos resultados obtidos, entre os indivíduos $\mathrm{HD}, \mathrm{HE}$ e $\mathrm{BH}$, significativa prevalência favorável aos indivíduos $\mathrm{HE}$ sobre os HD, sugerindo que nos mesmos, quando a comunicação não é realizada verbalmente e sim através de movimentos visuo-espaciais, com conotações emocionais, cuja competência é mais pertinente ao hemisfério direito, ainda assim, a LIBRAS apresenta maior incidência de processamento no hemisfério esquerdo, sinalizando que os aspectos lingüísticos predominam sobre os espaciais no aprendizado e compreensão da LIBRAS. Em relação ao $\mathrm{BH}$ e HE não ocorreu prevalência significativa.

Concluiu-se, também, após a comparação dos resultados obtidos entre os indivíduos $\mathrm{HD}, \mathrm{HE}$ e $\mathrm{BH}$, uma significativa prevalência favorável aos indivíduos HE sobre os HD, sugerindo que mesmo quando a comunicação não é realizada verbalmente e sim através de movimentos visuo-espaciais, com conotações emocionais, cuja competência é mais pertinente ao hemisfério direito, ainda assim, a LIBRAS apresenta maior incidência de processamento no hemisfério esquerdo, sinalizando que os aspectos lingüísticos predominam sobre os espaciais no aprendizado e compreensão da LIBRAS.

\section{REFERÊNCIAS BIBLIOGRÁFICAS}

BANICH, Marie T. Fundamentos da Neurociência. New York: Houghton Miffin Company, 1993. p 129-97.

BRODAL, A. Anatomia neurológica com correlações clínicas. 3 Ed.São Paulo: Roca, 1984.

CUPELLO, R.C.M. e MIRANDA, A. B. R. Rupturas em trajetos cerebrais subjacentes a alguns sinais neurolingüísticas encontrados em diversos tipos de afasia. Fono Atual. Rio de Janeiro, Ano 6 N 23. p. 42-59. 2003.

FAIRWEATHER, M.M. \& SIDAWAY, B. Estratégias pedagógicas hemisféricas utilizadas na aquisição e na retenção de uma habilidade motora. American Alliance for Health Physical Education, Recreation and Dance. Vol.65, 1995.

FERNANDES, V. Papel dos Hemisférios do Cérebro. Disponível em < http:// www. interFisio.com.Br> - 2000/ 2001. Acessado em 24/09/03.

MOURA, M.C.;LODI, A.C.; PEREIRA, M.C. Língua de sinais e educação do surdo. São Paulo: Sociedade Brasileira de Neuropsicologia (SBNp), 1993. 